\author{
Anna DASZKIEWICZ \\ Uniwersytet Gdański \\ daszkiewiczanna@gmail.com
}

\title{
ORTS- UND SITUATIONSGEBUNDENER SPRACHGEBRAUCH: ZUM GASTARBEITERDEUTSCH TÜRKISCHSTÄMMIGER MIGRANTINNEN UND ZU SEINER MEDIALEN REPRÄSENTATION
}

\section{GEGENSTAND UND ZIEL}

Ziel des vorliegenden Beitrags ist es, das Deutsch der ZuwandererInnen aus den 60er und 70er Jahren (insbesondere türkischer Herkunft) aufzuzeigen und $\mathrm{zu}$ charakterisieren, d.h. seine grundlegenden Merkmale in morphologisch-syntaktischer und lexikalischer Hinsicht in den Fokus zu stellen. Vorab sei angemerkt, dass im empirischen Teil des Beitrags die sekundäre (medial stilisierte) und nicht die ursprüngliche Form des Sprechstils ehemaliger GastarbeiterInnen untersucht wird. Als Untersuchungsmaterial gelten Ausschnitte aus den Filmproduktionen Kebab Connection (2004) ${ }^{1}$ und Almanya - Willkommen in Deutschland (2011) ${ }^{2}$. Obwohl die sprachlichen Besonderheiten im filmischen Kontext dicht angewandt sind und der Sprechstil der Protagonisten damit »verzerrt« wirkt,

1 Kebab Connection (2004): Regie (Anno Saul), Drehbuch (Ruth Toma, Fatih Akin, Jan Berger und Anno Saul), Besetzung (Güven Kıraç - Mehmet; Nursel Köse - Hatice; Denis Moschitto - Ibrahim; Nora Tschirner - Titzi; Hasan Ali Mete - Ahmet; Adnan Maral Kirianis). Die Deutschlandpremiere des Films fand am 30. Oktober 2004 statt.

2 Almanya - Willkommen in Deutschland (2011): Regie (Yasemin Şamdereli), Drehbuch (Yasemin und Nesrin Şamdereli), Besetzung (Vedat Erincin - Hüseyin; Fahri Ögün Yardim - der junge Hüseyin; Lilay Huser - Fatma; Demet Gül - die junge Fatma; Aykut Kayacik - Veli; Aycan Vardar - der junge Veli; Ercan Karacayli - Muhamed; Kaan Aydogdu - der junge Muhamed; Siir Elogdu - Leyla; Aliya Artuc - die junge Leyla; Petra Schmidt-Schaller - Gabi; Denis Moschitto - Ali; Aylin Tezel - Canan; Trystan Vyn Puetter - David; Rafael Koussouris - Cenk). Die Deutschlandpremiere des Films fand am 1. März 2011 in München statt. 
wird das »verfremdete« Deutsch aus Migrantenvierteln möglichst getreu wiedergegeben. Zumindest liefert der Film zahlreiche Verweise auf Abweichungen von dem Standarddeutschen, die laut linguistischen Studien das Sprachrepertoire der ersten Generation (hier: türkischstämmiger) ZuwanderInnen kennzeichnen.

Hierbei werden Pioniermigranten als Gastarbeiter und deren am Arbeitsplatz und im Alltag ungesteuert (ohne einen formalen Unterricht) erworbener kommunikativer Stil (»Sozialstil « ${ }^{3}$ als Gastarbeiterdeutsch (zum ersten Mal in einer Pilotstudie von Clyne [1968] beschrieben ${ }^{4}$ ) definiert. Bemerkenswert ist im Zusammenhang damit, dass diese Bezeichnung die bundesdeutsche Haltung gegenüber »Hinzugezogenen « offenlegt und ihr keine pejorativen Konnotationen anhaften sollten:

[Der Terminus] »Gastarbeiter « sagt etwas aus über bundesdeutsches Gastverständnis, er sagt etwas aus über das unverblümte Verhältnis vieler Menschen unserer Gesellschaft zu unseren ausländischen Mitbürgern. Schließlich - und das ist der gewichtigste Grund für seine Beibehaltung wurde er von denen, die so genannt werden, angenommen und wir ändern wenig an all seinen Konnotationen, solange sich nicht die pragmatischen Bedingungen seiner Verwendung ändern. Aber das läßt sich nicht durch ein einfaches Substitut beschönigen oder leugnen. (Hinnenkamp 1982: XVI)

Dennoch wird die Gastarbeiter-Figur in den 70er Jahren (und bis heute konsequent) in rein deutschen Medien vorwiegend als eine ausgebeutete Arbeitskraft dargestellt, was seinen Niederschlag jeweils in dem von der »deutschmonolingualen« Norm abweichenden Sprechstil findet.

3 Das Konzept des sozial kommunikativen Stils wurde von der Mannheimer Forschergruppe um Werner Kallmeyer, Inken Keim und Johannes Schwitalla erarbeitet (Kallmeyer [Hg.] 1994, 1995; Schwitalla 1995; Keim 1995). Demnach drückt der Stil die soziale und kulturelle Identität der Interaktanten aus (ihre Kommunikation erfolgt nie voraussetzungslos, sondern sie wird jeweils durch deren in die Interaktion mitgebrachtes Alltagsbzw. Hintergrundwissen beeinflusst), entscheidet über deren Positionierung in einer bestimmten sozialen Welt (vgl. Keim/Schütte 2002: 10). Hierbei wird unter dem Terminus Stil "die von Mitgliedern einer sozialen Einheit [...] getroffene Auswahl an und Weiterentwicklung von verbalen und nonverbalen Ausdrucksformen aus den ihnen zur Verfügung stehenden Ressourcen für die Selbstpräsentation und für die Durchführung kommunikativer Aufgaben" (Keim 2001: 376) verstanden.

4 Gemeint ist eine Pilotstudie, an der sich insgesamt 15 AusländerInnen mit spanischer, griechischer, türkischer und slowenischer Erstsprache beteiligt haben. Aufgrund ihrer Aussagen war es für Clyne möglich, ein Pidginvarietäten-Gefüge festzulegen, das Einwortsätze, Ausfall von Verben, Fehlen des bestimmten und unbestimmten Artikels, Ausfall von Flexionsformen, Verwendung des Infinitivs, Generalisierung des femininen Artikels 'die', Verwendung von 'viel' als Gradpartikel oder 'niks' als Wort- und Satznegation auszeichnen. 
Im Hinblick auf das medial konstruierte, verbreitete und popularisierte Gastarbeiter-Bild merken Dirim/Auer Folgendes an:

Sein typischer Vertreter ist der Türk 'Ali', dessen Rolle Günther Wallraff für seine Reporter-Tätigkeit als under cover-Journalist erfunden, selbst gespielt und später im Buch Ganz unten (1985) porträtiert hat: die Rolle des einfachen und ungebildeten, aber moralisch integreren Malochers in der Fabrik, der als Arbeitskraft ausgebeutet und als Türke von den Deutschen ausgegrenzt wird. An diesem Modell orientierte sich der linksliberale Diskurs in der Bundesrepublik, dessen Mitgefühl und soziales Engagement eben diese Rolle 'des Türken' zementierte. (Dirim/Auer 2004: 4, hervor. im Original)

Vor diesem Hintergrund verwundert die Tatsache, dass das Sprachrepertoire der Betroffenen im überwiegenden Maße nur wenig entwickelte Deutschvarietäten ausmachen, sowie dass die meisten von den Akteuren trotz eines mehrjährigen Aufenthalts in Deutschland auf einem defizitären Sprachstand geblieben sind, kaum. Zur Erläuterung des im Grunde auf rudimentären Sprachkenntnissen beruhenden Phänomens wird in der Sprachkontakt- und Zweitspracherwerbsforschung auf das Konzept der "lebensweltlichen Zweisprachigkeit”, das Ingrid Gogolin 1988 geprägt hat, zurückgegriffen. Gemeint ist der Zweitspracherwerb im kommunikativen Alltag und zwar in der Form, die die autochthone Sprachgemeinschaft vorgeschlagen und/oder dargeboten hat ${ }^{5}$ (,keine formal vermittelte, an kodifizierten Normen ausgerichtete Varietät, die nach standardsprachlichen Kriterien, d.h. nach Korrektheit und nach monolingualer Vollständigkeit zu beurteilen wäre" [Dirim/Auer 2004: 70]). Hierbei korreliert Gogolins Konzept stark mit der Pidgin ${ }^{6}$

5 Siehe hierzu (Limbach 2008: 53, hervor. von A.D.): „Was «die Türken» verstehen oder nicht verstehen, so belehrt uns Seyran Ateş, hänge davon ab, welches Bild man von den Zuwanderern habe und wie man mit ihnen spreche. Ihre Eltern hätten nur «Tarzandeutsch» lernen können, weil man mit ihnen nur so gesprochen habe. Nicht nur

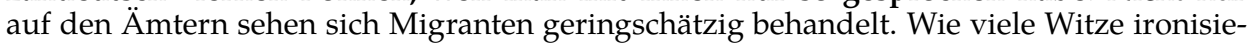
ren inzwischen die Tatsache, dass mit Migranten oder Menschen, die fremd wirken, ein verkümmertes, auf Infinitive verkürztes Deutsch gesprochen wird und diese gern geduzt werden."

6 Siehe hierzu (Riehl 2009: 122-123): „Tatsächlich weist das Deutsch der Gastarbeiter (der 60er und 70er Jahre) Züge eines Pidgins auf. Das gilt vor allem für das Prinzip der Simplifizierung, das ein Grundmerkmal von Pidginsprachen ist. So fallen etwa im Gastarbeiterdeutsch verschiedene syntaktische Kategorien (Artikel, Personalpronomina, Präpositionen, Kopula) weg, Verbformen stehen im Infinitiv, Flexionsendungen fehlen und es besteht eine Tendenz zur Generalisierung des femininen Genus (vgl. Meisel 1975: 21). Das Gastarbeiterdeutsch hat zudem mit Pidgins gemeinsam, dass es nur eingeschränkt ge- 
und foreigner-talk-These. Demnach stellen Pidgins vorwiegend situationsgebundene Kontaktsprachen dar, wo mittels einfachen und begrenzten Ausdrucksinventars Sprechakte realisiert werden. In Anbetracht der Tatsache, dass Pidgins grammatisch defekte Satzformen kennzeichnen, wird hier ein grammatisches Kontinuum durch Gestik und Mimik begleitet oder ergänzt. Die besagten Kontaktsprachen sind für Kommunikationsanforderungen in den klassischen Kolonialländern charakteristisch. Sie kamen dann zum Vorschein, wenn Sprecher anderer Erstsprachen aufeinander trafen und miteinander agieren mussten. Hierbei galten Arbeitssituationen in Handel, Landwirtschaft und Produktion als typische Kontexte (vgl. Keim 2012: 99). Aufgrund der Unkenntnis der Sprache der Aufnahmegesellschaft wurde ein stark reduziertes und auf den jeweiligen Arbeitskontext bezogenes Sprachkapital entwickelt, dessen Basis Wörter und grammatische Strukturen aus Zweit-/Herkunftssprachen jeweiliger Gesprächspartner stellten. Darauf weist Keim wie folgt hin:

In den klassischen Kolonialländern bildete die Sprache der sozial dominanten Gruppe, der Kolonialmacht, oft die Basis für das Pidgin, zumindest im Bereich des Wortschatzes. Im Bereich der Grammatik wurden die Strukturen aus den Sprachen übernommen, die für die beteiligten Sprecher einfach zu erlernen waren. Wenn z.B. eine Sprache ein komplexes morphologisches System hatte (Genus, Numerus, Kasus, Verbflexion etc.), die andere aber nicht, wurden die komplexen Formen vereinfacht oder weggelassen, z.B. Tempusformen auf Grundformen reduziert und Vor- und Nachzeitigkeit durch Zeitadverbien ausgedrückt. (Keim 2012: 99) ${ }^{7}$

In Anbetracht dessen sind Pidgins »Mischsprachen « und keine Erstsprachen einer bestimmten Sprechergemeinschaft, die aufgegeben werden können, sobald es an dem Anlass zu einer polylingual ausgerichteten Kommunikation fehlt. Nun aber können sie in über längere Zeiträume stabilen Kontaktsituationen zu voll ausgebauten Sprachsystemen (Kreolsprachen) und folglich auch (zu) Erstsprachen und Landessprachen evaluieren/aufsteigen (vgl. Keim 2012: 100). Dabei werden Ausbildung und Etablierung eines Pidgin durch den so genannten foreigner talk begünstigt (vgl. Meisel 1975: 41, Keim 2012: 100). Der Terminus foreigner talk referiert

braucht wird (nämlich am Arbeitsplatz) und auch als Verständigungssprache von Sprechern verschiedener nicht-deutscher Herkunftssprachen untereinander verwendet wird, z.B. von Gastarbeitern aus Italien, Griechenland und der Türkei."

7 Siehe dazu noch (Riehl 2009: 120-121). 
auf eine „spontane oder gewohnheitsmäßige Vereinfachung der eigenen Sprache in Anpassung an die [...] Erfordernisse der Kontaktsituation mit Anderssprachigen" (Bechert/Wildgen 1991), was der Linguist Hinnenkamp wie folgt argumentiert:

Sowohl im Deutschen als auch im Türkischen weichen Muttersprachler in bestimmten Situationen und gegenüber solchen Sprechern, die sich durch die Eigenschaft ausweisen, daß sie der Muttersprache der 'ingroup' gar nicht oder nur beschränkt mächtig sind bzw. deren Gebrauch ihnen aus den unterschiedlichsten Gründen als 'defizitär' unterstellt wird, von den üblichen standardisierten und umgangssprachlich akzeptierten Sprachvarianten der Sprachgemeinschaft ab. Das sprachliche Produkt dieser spezifischen Abweichung können wir als simplifiziert bezeichnen. Unterschiedliche Sprachgemeinschaften haben dafür ihre eigenen Etikette geprägt, wobei ich in Anlehnung an die traditionelle linguistische Bezeichnung, diese Sprechweise als 'Foreigner Talk' bezeichnet habe. Aus Gründen der Unterscheidung habe ich für den türkischen FT den ethnographischen Terminus Tarzanca bzw. Tarzanisch gewählt. (Hinnenkamp 1982: 154, hervor. im Original)

Obwohl im Gastarbeiterdeutsch Spezifika, die foreigner talk oder Tarzanca aufweisen, im Wesentlichen beibehalten werden, lassen sich manche Abweichungen vom Standarddeutschen als Interferenzerscheinungen ${ }^{8}$ (Übertragung erstsprachlicher Strukturen auf die Zielsprache [hier: auf die deutsche Standardsprache]) auslegen. Hierbei entpuppt sich das Übergeneralisierungs-Verfahren bei türkischstämmigen SprecherInnen vorwiegend als ein negativer Transfer: „Entgegen allen Vermutungen gibt es zwischen Deutsch und Türkisch - bis auf die lateinische Schreibweise und einen ähnlichen Buchstabenbestand - kaum Gemeinsamkeiten. Schwerwiegende Fehler sind somit vorprogrammiert" (Konyalioglu-Busch 2008: 5). Das rührt daher, dass Deutsch und Türkisch nicht nur verschiedenen Sprachtypen, sondern auch Sprachfamilien angehören, im Sprachtyp und in der Grammatik also weit auseinander liegen. Während das Deutsche wie das Englische in der germanischen Sprachfamilie verwurzelt ist und den flektierenden Typ repräsentiert, gehört das Türkische wie Kasachisch oder Azerbajdzhanisch zu der Familie

8 In Anbetracht der Tatsache, dass der Terminus Interferenz 'Einmischung' bedeutet und ihm daher negative Konnotationen anhaften, schlägt Clyne es vor, ihn durch den Begriff Transferenz im Sinne von 'Übertragung' oder 'Übernahme' zu ersetzen: „Transference is employed for the process of bringing over any items, features or rules from one language to another and for the results of this process. Any instance of transference is a transfer" (Clyne 1991: 160, hervor. im Original). 
der Turksprachen, für die der agglutinierende Typ charakteristisch ist. Demnach werden hier Deklination, Konjugation und Wortbildung durch Anfügen von Suffixen an den jeweiligen Wortstamm vollzogen. Wie bereits zuvor angedeutet, machen dem Standardtürkischen entlehnte grammatische Strukturen den Kern des Gastarbeiterdeutsch aus. Der besseren Übersichtlichkeit halber werden jetzt wesentliche Charakteristika des Standardtürkischen, die im gravierenden Kontrast zu sprachlichen Spezifika des Standarddeutschen stehen, aufgelistet und erläutert. Es sei ergänzend hinzugefügt, dass zum Erstellen einer im Grunde auf sprachlichen Gegensätzlichkeiten beruhenden Auflistung auf die Arbeiten von (Keim 1978), (Keim 2012), (Konyalioglu-Busch 2008) und (Hinrichs 2013) zurückgegriffen worden ist.

1. Der Clash zwischen Deutsch und Türkisch manifestiert sich bereits im Bereich der Phonetik: Im Türkischen gibt es nämlich „weder Diphthonge noch entsprechende Lautkombinationen wie /eu/, /au/ und dergleichen. Da Doppelkonsonanten fehlen, wundert es nicht, wenn aus »offen" nur »Ofen« wird. Fehler ergeben sich zusätzlich aus dem Alphabet und der entsprechenden Buchstaben-Laut-Kombination. Im Türkischen kennt man kein /ä/, nur /e/. [...] Die Buchstaben /q/, /w/, /x/, /B/ kommen nur in Fremdwörtern vor, dafür gibt es im Türkischen zusätzlich $/ \hat{\mathrm{g}} /$, /ş/, /ç/ und /1/. Da man also im Türkischen kein /w/ kennt und nur zwischen /v/ und /f/ unterschieden wird, kann es beispielsweise passieren, dass das »Wetter « $z u$ »Vetter « oder der »Vetter « als »fetter « bezeichnet wird" (Konyalioglu-Busch 2008: 5, hervor. im Original). Des Weiteren treten im Türkischen weder Doppelkonsonanten noch Doppelvokale auf, wie es bspw. bei deutschen Wörtern Aal, leer, Meer, Boote der Fall ist.

2. Darüber hinaus gehört Türkisch zu jenen Sprachen, in denen das Personalpronomen weggelassen werden kann, wenn es Subjekt ist (»Pro-dropSprache «): „Das Subjekt versteht sich quasi von selbst und die Person wird ohnehin hinten am Verb angezeigt: _ gidiyorum 〈ich gehe〉, _ unuttun 〈du hast [es] vergessen〉, also nicht: ben gidiyorum 〈ich ...〉 usw." (Hinrichs 2013: 75, hervor. im Original).

3. Im Gegensatz zum Deutschen, wo es vier Kasus gibt, stehen im Türkischen sechs Kasus zur Verfügung. Neben den auch im Deutschen präsenten Nominativ (wer/was?), Genetiv (wessen?), Dativ (wem?) und Akkusativ (wen/was?) gibt es den Lokativ (wo?) und den Ablativ (von wo?). Der Dativ dient auch zur Richtungsangabe (wohin?).

4. Im Türkischen gibt es keine Artikel.

5. Im Türkischen gibt es keine geschlechtliche Unterscheidung in der 3. Person Singular und nur eine einzige Personenbezeichnung: Statt er, sie, es benutzt man hier nämlich $o$. 
6. Im Türkischen gibt es in der 3. Person Singular nur ein Possessivpronomen onun.

7. Im Gegensatz zum Deutschen wird die türkische Plural-Form mittels der Suffixe -ler/-lar vollzogen, die jeweils an die Grundform des Nomens angehängt/angeklebt werden: 1) $e v=$ das Haus, bir ev = ein Haus ev-ler = die Häuser; 2) oda $=$ das Zimmer, bir od $a=$ ein Zimmer, oda-lar $=$ die Zimmer. Daraus ist ersichtlich, dass kein türkisches Wort durch das Anhängen von Suffixen verändert wird (vgl. dt. Streit - Streitereien/Streitigkeiten; Rat(schlag) - Ratschläge; Dank - Danksagungen etc.).

8. Auch die Deklination der Nomina erfolgt im Türkischen durch das Anhängen von Suffixen: Nominativ: $e v=$ das Haus, Genitiv: $e v-i n=$ des Hauses; Dativ: ev-e = dem Haus/nach Hause; Akkusativ: ev-i (das Haus); Lokativ: $e v-d e=$ in dem Haus; Ablativ: $e v$-den = von/aus dem Haus.

9. Ähnlich kennt man im Türkischen die Hilfsverben sein und haben nur als Endung.

10. Im Türkischen werden Präpositionen durch Postpositionen (nachgestellte Partikelwörter) oder durch Kasussuffixe ausgedrückt, bspw.: in meinem Haus = ev-im-de (Haus-mein-in), für Fatma = Fatma-için (Fatma-für).

11. Im Türkischen gibt es das Wort çok, das im Sinne von viel und sehr verwendet werden kann, bspw. çok güzel (sehr schön) und çok selamlar (viele Grüße). Bei der Verwendung von viel anstelle von sehr im Deutschen (als Gradpartikel) ist demnach eine direkte Übernahme aus dem Türkischen höchstwahrscheinlich.

12. Ebenso lassen sich analytische Verbbildungen, die im Gastarbeiterdeutsch vorhanden sind, wie bspw. kontroll machen und telefon machen als Übertragungen aus dem Türkischen erklären: telefon machen entspricht der türkischen Konstruktion telefon etmek (wörtlich: Telefon machen) und kontroll machen der türkischen Konstruktion kontrol etmek (wörtlich: Kontrolle machen).

13. Im Türkischen gibt es keine Unterscheidung zwischen schwachen und starken Verben sowie keine (Verb-)Präfixe wie bspw. miß, ver, zer, u.a.

14. Satzbau und Wortstellung: „Der türkische Satzbau unterscheidet sich grundsätzlich vom Deutschen, ebenso die Wortfolge, die im Standarddeutschen relativ festgelegt ist. Das flektierte Verb steht am Satzende [»Subjekt-Objekt-Verb«], davor kommen die Verbobjekte und davor steht das Subjekt. Das unbestimmte Akkusativobjekt steht direkt vor dem Verb, Lokal- oder Temporalangaben stehen vor dem Subjekt am Satzanfang. Ein einfacher türkischer Satz sieht also folgendermaßen aus: tk.: dün baba-m abla-m-a bir kitab getir-di (wörtl.: gestern Vater-mein Schwester-mein [Dat.] ein Buch gebracht hat) Dt.: gestern hat mein Vater meiner Schwester ein Buch gebracht" (Keim 2012: 94).

15. Im Gegensatz zum Deutschen gibt es im Türkischen keine Nebensatzkonstruktionen. Nebensätze werden im Türkischen durch Verbalsubstantive, Verbaladverbien und Partizipien ausgedrückt. 
Dass die oben präsentierten Besonderheiten des Standardtürkischen auf die Zielsprache übertragen werden (abfärben), geht aus der Charakteristik des Gastarbeiterdeutsch hervor. Demnach finden sich unter grundlegenden Merkmalen des Sprachrepertoires der PioniermigrantInnen (insbesondere türkischer Abstammung) Ausfall von Artikeln, Präpositionen, Pronomina, Kopulae; Ausfall bzw. Vereinfachung der Verbflexion (Numerus, Tempus, Modus, Person); übermäßige Verwendung des Infinitivs; Verbendstellung sowie Negationspartikel nix vor dem Verb. Zudem wirkt das Lexikon beschränkt, indem einzelne Sprachformen übergeneralisiert, Verben aufgespalten (telefon machen statt telefonieren [s. oben]), Vor- und Nachzeitigkeit durch Temporaladverbien ausgedrückt werden.

Auf das Faktum, dass das wenig entwickelte Deutsch Äußerungen von PioniermigrantInnen charakterisiert, wird in Arbeiten zu den türkischen GastarbeiterInnen genügend hingewiesen. Erwähnt seien an dieser Stelle die Veröffentlichungen des Heidelberger Forschungsprojekts „Pidgin-Deutsch” (1975), von Bodemann/Ostrow (1975), Meisel (1975), Keim (1978), Yakut (1981), Dittmar (1982), Keim/Nikitopoulos/Repp (1982), Hinnenkamp (1982), Becker/Klein (1984) oder Roche (1989), unter denen die Studie von Inken Keim (1978) immer noch als "die reichste Fundgrube" (Hinrichs 2013: 176) gilt. Da mit dem Anwerbestopp und sinkenden Immigrationszahlen türkischer ArbeiterInnen in den 80er Jahren das sprachwissenschaftliche Interesse an deren Sprechweise stark nachgelassen hat, ist der aktuelle Sprachstand dieser Sprechergruppe so gut wie nicht bekannt (vgl. Dirim/Auer 2004: 14). Abhilfe schaffen allerdings mediale Produkte (Konstrukte), die die Figuren von ehemaligen Gastarbeitern samt ihrem klischeebehafteten kommunikativen Sozialstil auf die Leinwand bannen. Wie eingangs angedeutet, dienen dem vorliegenden Beitrag die medialen Produktionen Kebab Connection (2004) und Almanya - Willkommen in Deutschland (2011) zur Erläuterung des besagten Sprachphänomens. Da die Nachvollziehbarkeit von sprachlichen Nuancen, kulturell bedingten Verhaltensweisen sowie deren Zielsetzung überhaupt jeweils der Situierung in Kontext und Situation bedarf, erfolgt an dieser Stelle eine kurze Charakteristik der Protagonisten (Gastarbeiter-Figuren). Im Nachhinein wird auf ihre Sprechweise fokussiert. 


\section{CHARAKTERISTIK DER GASTARBEITER-FIGUREN IM UNTERSUCHTEN FILMSTOFF}

Das Faktum, dass die „akute Not auf dem Arbeitsmarkt” 9 türkische Migrantenfamilien nach Deutschland trieb sowie dass sie den Aufenthalt in Almanya als vorübergehend betrachteten und dementsprechend in Stadtvierteln deutscher Großstädte (Köln und Hamburg) Enklaven bildeten, wo Türkisch dominant blieb und Deutsch nur bruchstückhaft gesprochen wurde, bringt die besagten Filmproduktionen auf einen gemeinsamen Nenner. Aufgrund des Anwerbeabkommens mit der Türkei, das am 31.10.1961 erfolgte, war es für Hüseyin Yilmaz ${ }^{10}$ und Mehmet Secmez ${ }^{11}$ sowie für viele ihrer Landsleute möglich, die alte Heimat zu verlassen und in dem Aufnahmeland eine angeblich sicherere Existenz aufzubauen. Demnach kam Hüseyin Yilmaz am 10.09.1964 auf dem deutschem Bahnhof Köln-Deutz an, und zwar am Tag, an dem die Zahl der Einwanderer einen historischen Rekord erreicht hat. Dass der Betroffene als einmillionunderste Gastarbeiter eine Anstellung im Aufnahmeland fand, hatte den Nachzug seiner Angehörigen sowie die Einbürgerung der ganzen Familie zur Folge. Dass die beiden Gastarbeiter-Figuren im Film (Hüseyin und seine Frau Fatma) nach dem 45-jährigen Aufenthalt in $\mathrm{Al}$ manya die deutsche Staatsangehörigkeit bekamen, bereitete dem Oberhaupt der Familie (Hüseyin) viele Sorgen. Doch im Inneren ist der Betroffene seiner alten Heimat treu geblieben. Daher hat er in der Türkei ein Haus gekauft und wünscht es sich, mit seinen Angehörigen eine Reise zu den Wurzeln zu unternehmen. Obwohl sich die restlichen Familienmitglieder zuvor dieser Entscheidung widersetzen, brachen sie schließlich in die Türkei auf.

Eine andere Gastarbeiter-Figur bildet Mehmet Secmez; ein türkischstämmiger Taxifahrer, der seit 30 Jahren mit seiner Familie in dem Hamburger Schanzenviertel lebt und sich furchtbar weigert, wirklich deutsch $\mathrm{zu}$ werden. Dass sein Sohn Ibrahim eine deutsche Freundin hat, bringt Mehmet zuerst nicht in Rage. Sobald sie aber schwanger wird, enterbt er seinen Sohn. Nun aber beeindruckt Mehmet inzwischen die selbstbewusste Haltung von Titzi, die sich für das Kind mit einem Migran-

\footnotetext{
9 (Almanya - Willkommen in Deutschland 2011: 01:50 min).

10 Die Gastarbeiter-Figur im Film Almanya - Willkommen in Deutschland (2011).

11 Die Gastarbeiter-Figur im Film Kebab Connection (2004).
} 
tenjugendlichen entscheidet. Daher beschließt er es, sich mit Ibrahim zu versöhnen und seiner künftigen Schwiegertochter in besonders schweren Schwangerschaftsmomenten zu Hilfe zu kommen.

Bemerkens- und erwähnenswert ist an dieser Stelle, dass in den beiden Filmproduktionen eine klischeebehaftete Vorstellung über türkischstämmige Migranten in den Vordergrund gestellt und im komischen Modus verarbeitet wird. Demnach werden insbesondere ältere männliche Türkenfiguren als patriarchal, zurückhaltend, engstirnig auf ihren Vorstellungen beharrend sowie sozial unbeholfen entworfen und so verbildlicht. Hierbei wird das vorstehend skizzierte Bild von männlichen türkischstämmigen Einwanderern der ersten Generation jeweils durch deren abweichenden Sprechstil authentisiert und »ikonisiert«/»zementiert«. Demnach stehen türkischstämmige Gastarbeiter-Figuren in deutschen Medien im sichtbaren Kontrast zu ihren 'Nachkommen', die sich meist im nativen Umgangsdeutsch ausdrücken und somit in die bundesdeutsche Gesellschaft »integriert « wirken. Kurzum werden türkischstämmige Protagonisten in medialen Konstrukten nach einem binären (alters- und sprachbezogenen) Oppositionsmodell gestaltet.

\section{DAS STILISIERTE GASTARBEITERDEUTSCH}

Es sei angemerkt, dass das Gastarbeiterdeutsch bei den bereits charakterisierten Protagonisten keine homogene Sprachform bildet, sondern mehr oder weniger vereinfachte (pidginisierte) Deutschvarietäten enthält. Dabei sind unterschiedliche Niveaus ihrer Sprachfertigkeit in der Zweitsprache auf sprachinterne (den Einfluss des Türkeitürkischen) sowie sprachexterne Faktoren (Alter, Eigenmotivation, Sozialintegration in die Aufnahmegesellschaft oder eher Widerstand gegen die Assimilation, Auswahl der Alltagssprache oder einen arbeitsbedingten Zweitspracherwerb) zurückzuführen. Dennoch macht das rudimentäre und defizitäre Deutsch der Betroffenen, das vielmehr einer in den ersten Erwerbsstadien fossilisierten Lernersprache entspricht, plausibel deutlich, dass sie in einfachen Tätigkeiten beschäftigt waren, wo nur Grundkenntnisse in der Zielsprache zur Kommunikation genügten; in Abschottung von der Aufnahmegesellschaft lebten und sich grundsätzlich mit der eigenen Ethnie identifizierten und ihre Existenz darin als gesichert ansahen. Ihr eingeschränktes Ausdrucksvermögen, das sich in zahlreichen und merklichen Abweichun- 
gen vom Standarddeutschen niederschlägt (also in Endkonsonantentilgung, bloßen Nominalphrasen, Ausfall von Personalpronomina und Präpositionen in Präpositionalphrasen, Fehlen von Kopulae, Abweichungen in der Genus- und Kasusflexion sowie der Verbstellung, aber auch Verwendung von Neuzugängen aus dem Türkischen ${ }^{12}$ ), wird unten ausführlich belegt, und zwar, durch eine entsprechende Kontextsituierung aus linguistischer Sicht beleuchtet. Vorweg sei aber darauf hingewiesen, dass nicht alle hier vorkommenden sprachlichen Stilisierungen inkorrekt oder defizitär sind. Einige sprachliche Gegebenheiten wie bspw. Klitisierung des unbestimmten und bestimmten Artikels („,dann ... 'tschuldigung!"), Endsilbenabschwächung (,Ich red kein Deutsch mit dir! Denn ich red nie Deutsch!") oder Personalpronomen bei Infinitivkonstruktionen im Singular (,'Sag du mir nicht, was ich tun soll!”) sind schon längst im Umgangsdeutsch präsent und zwar zu einer usuellen Norm aufgestiegen, ohne dabei unbedingt auf einen normfernen Charakter einer Ausdrucksweise verweisen zu müssen.

Die folgenden Tabellen veranschaulichen die Untersuchungsergebnisse:

TABELLE 1. Spezifika des Gastarbeiterdeutsch in Kebab Connection (2004)

\begin{tabular}{|c|c|c|}
\hline & $\begin{array}{l}\text { Gesprächsausschnitte aus dem Film } \\
\text { Kebab Connection }\end{array}$ & $\begin{array}{l}\text { Spezifika des Gastarbeiterdeutsch } \\
\text { in Kebab Connection }\end{array}$ \\
\hline 1. & $\begin{array}{l}\text { Der Vater von Ibo: Das ist meine Sohn, meine } \\
\text { Löwe! Bitte, ruf mal die Türkei an, erzähl allen, } \\
\text { Ibo steht bei uns in »Hürriyet«. } \\
\text { Die Schwester von Ibo: Nimmst du mich zur Os- } \\
\text { carverleihung? } \\
\text { Der Vater: Du kennst doch die Tochter von } \\
\text { Burhan? } \\
\text { Die Schwester von Ibo: Die mit der Hasenschar- } \\
\text { te? } \\
\text { Der Vater: Ayla! [...] Ihr Vater sagt, sie will dich } \\
\text { kennen lernen. [...] Was ist los mit dir? } \\
\text { Ibo: Baba... } \\
\text { Der Vater: Was? }\end{array}$ & $\begin{array}{l}\text { Abweichungen in der Genus- } \\
\text { flexion: meine Sohn, meine Löwe, } \\
\text { ein deutsche Kind, meine Herz, } \\
\text { eine »Papi« sein }\end{array}$ \\
\hline
\end{tabular}

12 In mehrsprachigen Kontexten wird der Wechsel von einer Sprache in die andere als Code-Switching bezeichnet. Dabei herrscht unter Sprach(kontakt)forschern die Uneinigkeit darüber, ob dieser Terminus auch Einwort-Varianten oder Teilsätze umfasst. Während die einen Linguisten wie bspw. (Myers-Scotton 2002: 153) Einzelwörter aus der anderen Sprache als Beispiel für das besagte Phänomen ansehen, tendieren die anderen dazu, einzeln vorkommende Entlehnungen nicht als Code-Switching, sondern eher als nonce-borrowing (Poplack 2004, MacSwan 2005: 7) oder 'Ad-hoc-Entlehnung(en)' bzw. 'Ad-hoc-Übernahme' (Riehl 2001: 61, Riehl 2009: 21) aufzufassen und zu bezeichnen. 


\begin{tabular}{|c|c|c|}
\hline & $\begin{array}{l}\text { Gesprächsausschnitte aus dem Film } \\
\text { Kebab Connection }\end{array}$ & $\begin{array}{c}\text { Spezifika des Gastarbeiterdeutsch } \\
\text { in Kebab Connection }\end{array}$ \\
\hline 1. & $\begin{array}{l}\text { Ibo: Du wirst Opa. Du wirst Oma. Und du wirst } \\
\text { Tante Ayla. } \\
\text { Ayla: Super! } \\
\text { Ibo: Titzi ist schwanger. } \\
\text { Der Vater: Was sage ich seit Geburt nun dir? } \\
\text { Hm? } \\
\text { Ibo: Mach 'nen Taxischein? } \\
\text { [Da gibt ihm sein Vater eine Ohrfeige.] } \\
\text { Die Mutter von Ibo: Mehmet! } \\
\text { Der Vater: Was sage ich seit Geburt nun dir? } \\
\text { Hm? Du kannst mit eine deutsche Mädchen } \\
\text { ausgehen. Du kannst mit eine deutsche } \\
\text { Mädchen einschlafen. Du kannst sogar mit eine } \\
\text { deutsche Mädchen aufwachen! Aber du darfst } \\
\text { sie niemals, niemals, niemals ... schwängern. } \\
\text { Und was machst du, Sohn eines Esels? Hm? } \\
\text { Du machst es trotzdem! Meine Herz! Welche } \\
\text { Schande! Raus! Verschwinde aus meine Haus! } \\
\text { Raus! Ein deutsche Kind! Es wird nie »Baba« } \\
\text { sagen! Du wirst eine »Papi« sein! } \\
\text { Ibo: Ich würde es deshalb nicht weniger lieben. } \\
\text { Der Vater: »Papi«! Es wird nie Sprache von dir } \\
\text { sprechen! Ich red kein Deutsch mit dir! Denn } \\
\text { ich red nie Deutsch! Und ich lebe seit } 30 \text { Jah- } \\
\text { ren hier! Du verdammter Mistkerl! Machst ein } \\
\text { Kind mit einer Ungläubigen! Einen gottlosen } \\
\text { Bastard! } \\
\text { Ibo: Vater! } \\
\text { Der Vater: Taki lma bana ulan! Nix Vater! } \\
\text { Du hast keine Vater! Und Erbe kriegst du } \\
\text { nix! Hm? } \\
\text { [Zu einem türkischen Ausßenstehenden]: } \\
\text { Mein Sohn macht Kind mit eine Deutsche! } \\
\text { Der Türke zur Erwiderung, die Hände zum } \\
\text { Himmel hebend: Allah!13 }\end{array}$ & $\begin{array}{l}\text { Abweichung in der Verbstel- } \\
\text { lung: Was sage ich seit Geburt } \\
\text { nun dir? statt Was sage ich dir } \\
\text { nun seit der Geburt? } \\
\text { Artikeltilgung: seit Geburt, } \\
\text { Sohn eines Esels, Erbe kriegst du } \\
\text { nix!, macht Kind mit eine Deut- } \\
\text { sche } \\
\text { Negationspartikel nix anstelle } \\
\text { von kein(e) und nicht: Nix Va- } \\
\text { ter!, Erbe kriegst du nix } \\
\text { Ausfall von Personalprono- } \\
\text { mina und Verbspitzenstel- } \\
\text { lung: Machst ein Kind mit einer } \\
\text { Ungläubigen! } \\
\text { Türkische Ausdrücke mitten } \\
\text { im deutschen Satz: Es wird } \\
\text { nie »Baba« sagen!, Taki lma bana } \\
\text { ulan! } \\
\text { Zugänge aus dem Türkischen: } \\
\text { Baba (= Vater), Taki lma bana } \\
\text { ulan! (= Du hast keinen Vater } \\
\text { [mehr]!) }\end{array}$ \\
\hline 2. & $\begin{array}{l}\text { Der Vater von Ibo: Was für ein Hurricane! (hur- } \\
\text { ricane = Wirbelsturm) } \\
\text { Die Mutter von Ibo: Wir hätten sie reinbitten } \\
\text { sollen. } \\
\text { Der Vater von Ibo: Hab ich doch! Allah! Allah! }\end{array}$ & $\begin{array}{l}\text { Ausfall von Possessivprono- } \\
\text { mina: Sie hat Stolz statt Sie hat } \\
\text { ihren Stolz }\end{array}$ \\
\hline
\end{tabular}

13 (Kebab Connection 2004: 16:40-19:10 min). 


\begin{tabular}{|c|c|c|}
\hline & $\begin{array}{l}\text { Gesprächsausschnitte aus dem Film } \\
\text { Kebab Connection }\end{array}$ & $\begin{array}{l}\text { Spezifika des Gastarbeiterdeutsch } \\
\text { in Kebab Connection }\end{array}$ \\
\hline 2. & $\begin{array}{l}\text { Sie hat Stolz! Wie ein Mädchen aus Trabzon. } \\
\text { Die Mutter: Hübsch ist sie auch. } \\
\text { Der Vater: Für eine Deutsche. Warum war sie so } \\
\text { wütend auf Ibo? } \\
\text { Die Mutter: Dein Sohn hat sie verlassen. } \\
\text { Der Vater: Ach, was bleibt ihm anderes übrig? } \\
\text { Die Mutter: Mehmet! } \\
\text { Der Vater: Was, Mehmet? } \\
\text { Die Mutter: Bring mich nicht auf die Palme! Sie } \\
\text { ist alleine! Sie kriegt ein Baby! In meiner Familie } \\
\text { lässt ein Mann seine Frau nicht im Stich, wenn } \\
\text { sie schwanger ist. } \\
\text { Der Vater: Ja, in meiner Familie auch nicht! } \\
\text { Die Mutter: Dann unternimm was! Üff! Üff! } \\
\text { Der Vater: Sag du mir nicht, was ich tun soll! }{ }^{14}\end{array}$ & $\begin{array}{l}\text { Personalpronomen bei Infi- } \\
\text { nitivkonstruktionen im Sin- } \\
\text { gular: Sag du mir nicht, was ich } \\
\text { tun soll! statt Sag mir nicht, was } \\
\text { ich tun soll! }\end{array}$ \\
\hline 3. & $\begin{array}{l}\text { [In der Werkstatt] } \\
\text { Der Vater betritt den Raum und äußert sich zum } \\
\text { Sohn wie folgt: Warum lässt du deine schwange- } \\
\text { re Frau im Stich? Schäm dich! } \\
\text { Ibo: Du hast mich wegen Titzi verstoßen. } \\
\text { Der Vater: Dich schon, aber nicht meinen Enkel. } \\
\text { Ibo: Augenblick! Du wirfst mich raus, enterbst } \\
\text { mich, bist nicht mehr mein Vater, weil ich } \\
\text { 'n Kind mit 'ner Deutschen krieg'! } \\
\text { Der Vater: Ja. } \\
\text { Ibo: Ich streite mich deshalb mit Titzi, jetzt } \\
\text { wirfst du mir das vor? } \\
\text { Der Vater: Ja! } \\
\text { Ibo: Baba, das ergibt doch gar keinen Sinn! } \\
\text { Der Vater: So redest du nicht mit deinem Vater! } \\
\text { Der nicht mehr dein Vater ist! Eşoğlueşek! (aus- } \\
\text { gesprochen: escholeschek, ein türkisches Schimpf- } \\
\text { wort, das einen Eselssohn bedeutet) } \\
\text { Ibo: Die spinnen, die Türken!15 }\end{array}$ & $\begin{array}{l}\text { Türkischer Ausdruck mitten } \\
\text { im deutschen Satz: Eşoğlueşek! }\end{array}$ \\
\hline 4. & $\begin{array}{l}\text { [Auf der Straße treffen Titzi und der Vater von } \\
\text { Ibo abrupt aufeinander] } \\
\text { Der Vater von Ibo: Und Ibrahim? } \\
\text { Titzi: Ich hab 'nen Brief von ihm bekommen. } \\
\text { [Dann öffnet Titzi den Brief] } \\
\text { "Hiermit bestätigen wir Herrn Ibrahim Secmez } \\
\text { die erfolgreiche Teilnahme an unserem Vorberei- } \\
\text { tungskurs für schwangere Frauen." } \\
\text { Der Vater: Sohn eines Esels! }\end{array}$ & $\begin{array}{l}\text { Artikeltilgung: Sohn eines } \\
\text { Esels } \\
\text { Abweichung in der Verbstel- } \\
\text { lung: Wenn Sie brauchen Hilfe, } \\
\text { dann [...] statt Wenn Sie Hilfe } \\
\text { brauchen, dann [...] }\end{array}$ \\
\hline
\end{tabular}

14 (Kebab Connection 2004: 34:50-35:40 min).

15 (Kebab Connection 2004: 38:15-38:57 min). 


\begin{tabular}{|c|c|c|}
\hline & $\begin{array}{l}\text { Gesprächsausschnitte aus dem Film } \\
\text { Kebab Connection }\end{array}$ & $\begin{array}{c}\text { Spezifika des Gastarbeiterdeutsch } \\
\text { in Kebab Connection }\end{array}$ \\
\hline 4. & $\begin{array}{l}\text { Titzi: Wie bitte? } \\
\text { Der Vater: Ich sagte Sohn eines Esels. [...] Wenn } \\
\text { Sie brauchen Hilfe, dann ... 'tschuldigung! }{ }^{6}\end{array}$ & \\
\hline 5. & $\begin{array}{l}\text { [Im Krankenhaus] } \\
\text { Ibo zum Vater: Was machst du denn hier? } \\
\text { Der Vater: Ich mach deine Job! Warum bist du } \\
\text { nicht bei deiner Frau? } \\
\text { Titzi: Raus! Raus! Alle beide! }{ }^{17}\end{array}$ & $\begin{array}{l}\text { Abweichung in der Genus- } \\
\text { und Kasusflexion: Ich mach } \\
\text { deine Job! statt Ich mache deinen } \\
\text { Job! } \\
\text { Endsilbenabschwächung: Ich } \\
\text { mach statt Ich mache }\end{array}$ \\
\hline
\end{tabular}

TABELLE 2. Spezifika des Gastarbeiterdeutsch in Almanya - Willkommen in Deutschland (2011)

\begin{tabular}{|c|c|c|}
\hline & $\begin{array}{l}\text { Gesprächsausschnitte aus dem Film } \\
\text { Almanya - Willkommen in Deutschland }\end{array}$ & $\begin{array}{c}\text { Spezifika des Gastarbeiterdeutsch } \\
\text { in Almanya - Willkommen } \\
\text { in Deutschland }\end{array}$ \\
\hline 1. & $\begin{array}{l}\text { [Bei einem Familientreffen. Die ganze Familie } \\
\text { sitzt am Tisch.] } \\
\text { Hüseyin: Familie, ich habe Überraschung. } \\
\text { Fatma [unterbricht ihren Mann voller Freude]: } \\
\text { Wir sind jetzt Deutsche [und zeigt auf die vor } \\
\text { kurzem abgeholten deutschen Pässe]. } \\
\text { Hüseyin: Nein, das nicht Überraschung. } \\
\text { Überraschung ist, ich habe Haus gekauft. In } \\
\text { Türkei. Im Dorf. In Heimat. } \\
\text { Muhamed: Warum das denn? Wollt ihr jetzt } \\
\text { zurückkehren oder was? } \\
\text { Hüseyin zu Cenk [zeigt auf ein altes Foto, das } \\
\text { ein anatolisches Gehöft abbildet]: Schau mal, das } \\
\text { ist unser Heimat. Schön, ne? } \\
\text { Cenk: Da kommen wir her? } \\
\text { Hüseyin: Hm... [wandelt unerwartet ins } \\
\text { Türkische]. } \\
\text { Also, ich möchte, dass wir in den kommen- } \\
\text { den Ferien alle zusammen in die Türkei fah- } \\
\text { ren. Das Haus ist alt und muss renoviert wer- } \\
\text { den. Es wäre sehr gut, wenn ihr alle mithelfen } \\
\text { würdet. [...] Es reicht! Ruhe, verdammt noch } \\
\text { mal! Habe ich als euer Vater euch jemals um }\end{array}$ & $\begin{array}{l}\text { Artikeltilgung: Familie, ich habe } \\
\text { Überraschung,Überraschung ist, } \\
\text { ich habe Haus gekauft, in Türkei, } \\
\text { in Heimat } \\
\text { Abweichung in der Genusfle- } \\
\text { xion: unser Heimat } \\
\text { Kopulatilgung, gepaart mit } \\
\text { der falschen Negationsparti- } \\
\text { kel: das nicht Überraschung statt } \\
\text { das ist keine Überraschung }\end{array}$ \\
\hline
\end{tabular}

16 (Kebab Connection 2004: 59:15-1:00:22 $\mathrm{min}$ ).

17 (Kebab Connection 2004: 1:18:43-1:18:53 min). 


\begin{tabular}{|c|c|c|}
\hline & $\begin{array}{l}\text { Gesprächsausschnitte aus dem Film } \\
\text { Almanya - Willkommen in Deutschland }\end{array}$ & $\begin{array}{c}\text { Spezifika des Gastarbeiterdeutsch } \\
\text { in Almanya - Willkommen } \\
\text { in Deutschland }\end{array}$ \\
\hline 1. & $\begin{array}{l}\text { was gebeten? Ihr sitzt hier nur rum und macht } \\
\text { blabla... Schämt euch! Wir sind eine Familie [er } \\
\text { wechselt prompt ins Deutsche]... eine türkische } \\
\text { Familie! [Bir Türk ailesiyiz!] }\end{array}$ & \\
\hline 2. & $\begin{array}{l}\text { Auf die Frage von Fatma, warum ihr Enkel von } \\
\text { der Schule mit einem blauen Auge kommt, ant- } \\
\text { wortet seine Mutter Gabi: „Der türkische Junge } \\
\text { aus seiner Klasse hat ihn gehänselt, er sei nicht } \\
\text { der richtige Türke." }{ }^{9} \text { Da rastet Hüseyin aus und } \\
\text { richtet an Cenks Vater und seinen jüngsten Sohn } \\
\text { Ali die folgenden Worte: „Was? Du gehst mor- } \\
\text { gen zur Schule und zeigst diesem eşoğlueşek } \\
\text { (ausgesprochen: escholeschek), was für Türken wir } \\
\text { sind." } 21\end{array}$ & $\begin{array}{l}\text { Imperativ falsch ausgedrückt: } \\
\text { Du gehst morgen zur Schule und } \\
\text { zeigst diesem eşoğlueşek, was für } \\
\text { Türken wir sind statt Geh mor- } \\
\text { gen zur Schule und zeig diesem } \\
\text { eşoğlueşek, was für Türken wir } \\
\text { sind } \\
\text { Türkischer Ausdruck mitten } \\
\text { im deutschen Satz: eşoğlueşek }\end{array}$ \\
\hline 3. & $\begin{array}{l}\text { [Eine Szene in der Türkei. Hüseyin trainiert sei- } \\
\text { ne Rede, die er vor der deutschen Bundeskanz- } \\
\text { lerin A. Merkel halten sollte.]: „Hey, Angela! } \\
\text { Wo Problem? Du kommst aus dem Osten, ich } \\
\text { auch. Wir beide Ossis.”22 }\end{array}$ & $\begin{array}{l}\text { Verwendung des Aufmerk- } \\
\text { samkeitsmarkers hey zu Be- } \\
\text { ginn von Redebeiträgen: Hey, } \\
\text { Angela! } \\
\text { Ausfall von Artikeln, Prono- } \\
\text { mina und Kopulae: Wo Pro- } \\
\text { blem? Du kommst aus dem Os- } \\
\text { ten, ich auch. Wir beide Ossis. }\end{array}$ \\
\hline
\end{tabular}

\section{ABSCHLIEßENDE BEMERKUNGEN}

Im vorliegenden Beitrag wird dem entfremdeten Sprachgebrauch türkischstämmiger (männlicher) Pioniermigranten, dem Gastarbeiterdeutsch Rechnung getragen. Hierbei hat sich der besagte Sprech- und Kommunikationsstil unter spezifischen, denn mit der Migration einhergehenden Lebens- und Arbeitsbedingungen herausgebildet. Da die Arbeitsmigration in die Bundesrepublik Deutschland nach dem zweiten Weltkrieg als eine vorübergehende Erscheinung wahrgenommen wur-

\footnotetext{
18 (Almanya - Willkommen in Deutschland 2011: 10:58-12:42 min).

19 (Almanya - Willkommen in Deutschland 2011: 09:35-09:39 min).

20 Ein türkisches Schimpfwort, das einen Eselssohn bedeutet.

21 (Almanya - Willkommen in Deutschland 2011: 09:40-09:47 min).

22 (Almanya - Willkommen in Deutschland 2011: 59:30-59:40 min).
} 
de, wurden von der Aufnahmegesellschaft rudimentäre und defizitäre Varietäten des Deutschen geduldet und sogar selbst in Gang gesetzt ('provoziert'). Darauf ist eine sozial symbolische Funktion des Gastarbeiterdeutsch zurückzuführen. Demnach gilt die fossilisierte Lernersprache der ersten Gastarbeitergeneration als Ausdruck sozialer Eigenschaften und Handlungsweisen: Sie liefert einen zuverlässigen Beleg für die ablehnende Haltung gegenüber Minderheitengruppen und zugleich für eine Art Distanz zur deutschen Mehrheitsgesellschaft. Demnach wird mittels des Gastarbeiterdeutsch die Kategorie des „,zurückgebliebenen Türken” realisiert, "der orientiert an den Traditionen seiner Herkunftsregion (wie er sie vor vielen Jahren erlebte) und darin verharrend, sich schwer tut, sich neuen Lebensumständen und -anforderungen gegenüber $\mathrm{zu}$ öffnen" (Keim 2002: 155). Andererseits wird mithilfe des Gastarbeiterdeutsch die Kategorie des „vorurteilsbehafteten Deutschen” aufgegriffen, „der Ausländer vor allem als 'grobe und ungebildete Gastarbeiter' sieht und behandelt" (Keim 2002: 155, hervor. im Original).

Da es derzeit an wissenschaftlicher Auswertung des kommunikativen Sozialstils ehemaliger GastarbeiterInnen und seiner Funktion mangelt, dienen mediale Konstrukte (vorwiegend mit einem unterhaltsamen Charakter) als empirische Datensätze und analytischer Leitfaden. Selbst wenn Spezifika des Gastarbeiterdeutsch im komischen Modus dicht angewandt und übersteigert wirken, korrelieren sie stark mit dem authentischen Sprechstil der Betroffenen. Daher wird im vorliegenden Beitrag zur Darstellung und Erläuterung des besagten Sprachphänomens auf die Filmproduktionen Kebab Connection (2004) und Almanya - Willkommen in Deutschland (2011) zurückgegriffen. Kurzum wird hier der Sprachstoff und genauer gesagt, der von der monolingualen Norm abweichende Sprechstil ehemaliger (türkischstämmiger) Gastarbeiter rekonstruiert und aus linguistischer Perspektive beleuchtet.

\section{BIBLIOGRAFIE}

Bechert, Johannes/Wildgen, Wolfgang (1991): Einführung in die Sprachkontaktforschung. Unter Mitarbeit von Christoph Schröder. Darmstadt: Wissenschaftl. Buchges.

Becker, Angelika/Klein, Wolfgang (1984): Notes on the internal organisation of a learner variety. In: Auer, Peter/di Luzio, Aldo (Hg.): Interpretive Soziolonguistics. Tübingen: Narr, 215-232. 
Bodemann, Y.M/Ostrow, R. (1975): Lingua franca und Pseudo-Pidgin in der Bundesrepublik: Fremdarbeiter und Einheimische im Sprachzusammenhang. In: LiLi 5 (17-19/20), 122-146.

Clyne, Michael (1968): Zum Pidgin-Deutsch der Gastarbeiter. In: Zeitschrift für Mundartforschung 35, 130-139.

Clyne, Michael (1991): Community Languages: The Australian Experience. Cambridge: CUP.

Dirim, İnci/Auer, Peter (2004): Türkisch sprechen nicht nur die Türken. Über die Unschärfebeziehung zwischen Sprache und Ethnie in Deutschland. Berlin: Walter de Gruyter

Dittmar, Norbert (1982): "Ich fertig arbeite - nicht mehr spreche Deutsch." Semantische Eigenschaften pidginisierter Lernervarietäten des Deutschen. In: LiLi 45, 84-103.

Gogolin, Ingrid (1988): Erziehungsziel Zweisprachigkeit. Konturen eines sprachpädagogischen Konzepts für die multikulturelle Schule. Hamburg: Bergman \& Helbig.

Heidelberger Forschungsprojekt „Pidgin-Deutsch” (1975): Sprache und Kommunikation ausländischer Arbeiter. Kronberg/Taunus: Scriptor Verlag.

Hinnenkamp, Volker (1982): Foreigner Talk und Tarzanisch. Eine vergleichende Studie über die Sprechweise gegenüber Ausländern am Beispiel des Deutschen und des Türkischen. Hamburg: Helmut Buske Verlag.

Hinrichs, Uwe (2013): Multi Kulti Deutsch. Wie Migration die deutsche Sprache verändert. München: C.H. Beck Verlag.

Jakobs, Eva-Maria/Rothkegel, Annely (Hg.) (2001): Perspektiven auf Stil. (= Reihe Germanistische Linguistik 226). Tübingen.

Kallmeyer, Werner (1994): Kommunikation in der Stadt. Teil 1: Exemplarische Analysen des Sprachverhaltens in Mannheim. (= Schriften des Instituts für deutsche Sprache 4.1). Berlin.

Kallmeyer, Werner (1995): Kommunikation in der Stadt. Teil 2: Ethnographien von Mannheimer Stadtteilen. (= Schriften des Instituts für deutsche Sprache 4.2). Berlin.

Keim, Inken (1995): Kommunikation in der Stadt. Teil 3: Kommunikative Stilistik einer sozialen Welt "kleiner Leute" in der Mannheimer Innenstadt. (= Schriften des Instituts für deutsche Sprache 4.3). Berlin.

Keim, Inken (1978): Gastarbeiterdeutsch. Untersuchungen zum sprachlichen Verhalten türkischer Gastarbeiter. Tübingen: Narr.

Keim, Inken (2001): Die Powergirls - Aspekte des kommunikativen Stils einer Migranntinnengruppe in Mannheim. In: Jakobs/Rothkegel (Hg.), S. 375-400.

Keim, Inken (2002): Bedeutungskonstitution und Sprachvariation. Funktionen des 'Gastarbeiterdeutsch' in Gesprächen jugendlicher MigrantInnen. In: Deppermann, Arnulf: Be-deuten: Wie Bedeutung im Gespräch entsteht. Tübingen: Stauffenburg-Verlag, 134-157.

Keim, Inken (2012): Mehrsprachige Lebenswelten. Sprechen und Schreiben der türkischstämmigen Kinder und Jugendlichen. Tübingen: Narr Verlag. 
Keim, Inken/Nikitopoulos, Pantelis/Repp, Michael (1982): Kommunikation ausländischer Arbeiter. Eine Studie zum deutschsprachigen Interaktionsverhalten von griechischen und türkischen Arbeitern. Tübingen: Narr.

Keim, Inken/Schütte, Wilfried (2002): Einleitung. In: Keim, Inken/Schütte, Wilfried (Hg.): Soziale Welten und kommunikative Stile: Festschrift für Werner Kallmeyer zum 60. Geburtstag. (= Studien zur Deutschen Sprache 22). Tübingen, S. 9-26.

Konyalioglu-Busch, Zerrin (2008): Deutsch als Zweitsprache - Türkische Schüler systematisch fördern. Buxtehude: Persen Verlag.

Limbach, Jutta (2008): Hat Deutsch eine Zukunft? Unsere Sprache in der globalisierten Welt, München: C. H. Beck Verlag.

MacSwan, Jeff (2005): Codeswitching and generative grammar. A critique of the MLF model and some remarks on 'modified minimalism'. In: Bilingualism: Language and Cognition 8, 1-22.

Meisel, Jürgen (1975): Ausländerdeutsch und Deutsch ausländischer Arbeiter. Zur möglichen Entstehung eines Pidgin. In: Zeitschrift für Literaturwissenschaft und Linguistik (LiLi), Heft 18, 9-53.

Myers-Scotton, Carol (2002): Contact Linguistics. Bilingual Encounters and Grammatical Outcomes. Oxford/New York: Oxford University Press.

Poplack, Shana (2004): Code-Switching. In: Ammon at all (Hgg.), 589-596.

Riehl, Claudia Maria (2001): Schreiben, Text und Mehrsprachigkeit. Zur Textproduktion in mehrsprachigen Gesellschaften am Beispiel der deutschsprachigen Minderheiten in Südtirol und Ostbelgien. Tübingen: Stauffenburg.

Riehl, Claudia Maria (2009): Sprachkontaktforschung: Eine Einführung. Tübingen: Narr.

Roche, Jörg (1989): Xenolekte: Struktur und Variation im Deutsch gegenüber Ausländern. Berlin: de Gruyter.

Schwitalla, Johannes (1995): Kommunikation in der Stadt. Teil 4: Kommunikative Stilistik zweier sozialer Welten in Mannheim-Vogelstang. (= Schriften des Instituts für deutsche Sprache 4.4). Berlin.

Yakut, Atilla (1981): Sprache der Familie. Eine Untersuchung des Zweitsprachenerwerbs der türkischen Gastarbeiterfamilien in der BRD. Tübingen: Narr.

ORTS- UND SITUATIONSGEBUNDENER SPRACHGEBRAUCH: ZUM GASTARBEITERDEUTSCH TÜRKISCHSTÄMMIGER MIGRANTINNEN UND ZU SEINER MEDIALEN REPRÄSENTATION

\section{Zusammenfassung}

Der vorliegende Artikel bringt einen Sprechstil heutiger DeutschländerInnen und zugleich türkischstämmiger GastarbeiterInnen, die in den 60ger und 70ger Jahren am meisten den deutschsprachigen Raum besiedelt haben, näher. Ihr 
"Gastarbeiterdeutsch", worauf deutlich die Fachliteratur zum besagten Gegenstand verweist, greift das Thema von „Pidgin”, „Foreigner-Talk” und der "linguistischen Interferenz" auf. Der empirische Stoff ist ein Filmmaterial, genauer gesagt die Sprache, die die Hauptprotagonisten in den Filmen Kebab Connection (2004) und Almanya - Willkommen in Deutschland (2011) benutzen. Mediale Stilisierung von "Gastarbeiterdeutsch" weist auf seine Abweichungen vom Standarddeutsch hin, auf das defizitäre Deutsch ehemaliger GastarbeiterInnen sowie die immer noch in der deutschen Gesamtbevölkerung bestehenden Stereotype.

Stichwörter: Sprechstil türkischstämmiger GastarbeiterInnen, die deutsche Standardsprache, die türkische Standardsprache, eine sprachliche Interferenz, mediale Stilisierung(en), die Sprache in den Medien, ethnische Vorurteile in Bezug auf die Sprache

\section{UŻYCIE JĘZYKA UWARUNKOWANE MIEJSCEM I SYTUACJĄ: O JĘZYKU NIEMIECKIM „GASTARBEITERÓW” POCHODZENIA TURECKIEGO I JEGO MEDIALNEJ KREACJI}

\section{Streszczenie}

Niniejszy artykuł przybliża język dzisiejszych obywateli Niemiec i zarazem tureckich emigrantów zarobkowych, którzy w latach 60. i 70. ubiegłego wieku najliczniej zaludnili niemieckie aglomeracje miejskie. Ich "Gastarbeiterdeutsch", na co wyraźnie wskazuje literatura przedmiotu, dobrze wpisuje się w kontekst zagadnień językowych jak „pidgin”, ,foreigner-talk” oraz „interferencji językowej". Materiał empiryczny to materiał filmowy, a zatem język, jakiego używają bohaterowie filmów Kebab Connection (2004) i Almanya - Willkommen in Deutschland (2011). Medialne stylizacje "Gastarbeiterdeutsch" wskazują wyraźnie na jego rozbieżności ze standardowym językiem niemieckim, demaskując przy tym językowy deficyt dawnych "gastarbeiterów”, a także obecne w narodzie niemieckim uprzedzenia etniczne.

Słowa kluczowe: język tureckich „gastarbeiterów”, standardowy język niemiecki, standardowy język turecki, interferencja językowa, medialne stylizacje języka, język w mediach, uprzedzenia etniczne w kontekście języka

\section{THE USE OF LANGUAGE RELATING TO PLACE AND SITUATION: ON THE 'GASTARBARBEITER' GERMAN OF MIGRANTS WITH TURKISH ORIGIN AND ITS MEDIA MANIFESTATION}

\section{Summary}

This article addresses the issue of the language of the first generation of German migrants (with Turkish origin), pointing to the conditions/circumstances 
of its formation. It presents an asymmetry between the standard Turkish and German languages, which is the main cause of mistakes resulting from interference (transference). The study is based on the film productions Kebab Connection (2004) and Almanya-Willkommen in Deutschland (2011), where although the gastarbeiter German is stylised, it is yet exemplary for the language forms discussed here.

Key words: gastarbeiter German language, pidgin, foreigner talk, bilingualism determined by life situation (place of residence), asymmetry between the Turkish and German languages, transference/interference 